\title{
Thermal Microwave Radiation for Subsurface Absolute Temperature Measurement
}

\author{
W. SUSEK \\ Institute of Radioelectronics, Faculty of Electronics, Military University of Technology \\ S. Kaliskiego 2, 00-908 Warsaw, Poland
}

\begin{abstract}
Problems related to thermal radiation of human bodies in the microwave range are presented in the paper from medical diagnosis point of view. The paper reviews physical principles of thermal radiation measurement at microwave frequencies. A design of a modulation type radiometer with both zero reading and compensation of influence of the reflectivity is presented in the paper. On the base of this design, requirements for absolute temperature measurement conditions in the lossy and homogeneous medium were found. It is worth noting that these demands do not need any limitations for environmental parameters, especially for their stability during measurement (ambient temperature stability). At the end, experimental results of the measurements carried out with the use of the microwave radiometer operating in the $1.5 \mathrm{GHz}$ band are shown.
\end{abstract}

PACS numbers: 87.63.Hg, 44.40.+a, 84.40.Dc, 07.57.Hm

\section{Introduction}

Radiometry is a technique, which is aimed at measurements of emitted radiation. Among these measurements noise radiometry [1] can be distinguished which is related to electromagnetic noise radiation emitted by all bodies with temperatures higher than $0 \mathrm{~K}$. If energy of a body is sufficiently high (i.e. its temperature is high enough) then emitted radiation is observed directly as light or heat. Bodies with lower temperatures like flat-iron or human body emit infrared radiation, while bodies with very low temperatures emit radiation in the radiowave range. In the microwave range depth of radiation penetration into object interior is much higher than for infrared radiation. That is why, a reception of thermal radiation originating from the investigated object inside using a fully non-invasive technique is pretty possible. New opportunities appeared due to applications of microwave range in noise radiometry. Among them the most promising and perspective are: medical diagnosis and therapy in which a radiometer is used for temperature control in hypo- and hyperthermia $[2,3]$, monitoring of pathology conditions of internal organs [4-8], up to detection of the carcinoma of the breast [9-11].

\section{Physical principles}

Physical premises for the occurrence of noise radiation are based on fundamental relations describing mutual interaction between charged elementary particles and energy. This interaction causes a change of energy state of the charged particle which, in turns, leads to emission of electromagnetic wave. Charged particles are a base of a structure of any physical body. These bodies, existing in temperatures higher than absolute zero temperature, accumulate and emit energy in the form of electromagnetic waves. Temperature of a body exactly determines power of this emission. In order to characterize the spectral dis-

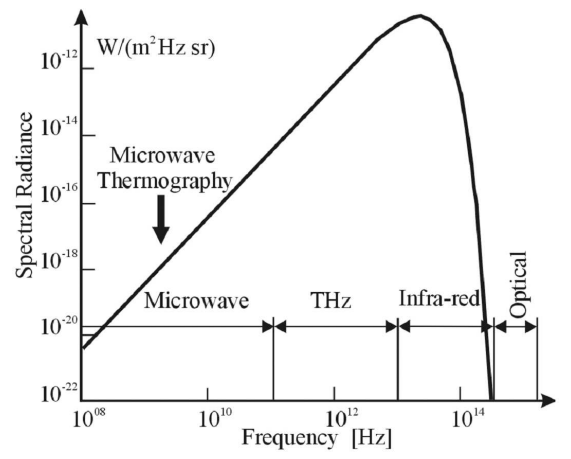

Fig. 1. Black body radiation for $T=290 \mathrm{~K}$.

tribution of thermal radiation a spectral emissivity $E_{f, T}$ is defined. In numbers, it equals the ratio of the energy $\mathrm{d} W$ radiated by unit square in a unit time in the form of electromagnetic wave in a narrow frequency band to the length of this band. The $E_{f, T}$ value depends on frequency, temperature, chemical composition of the body, and a state of its surface. Max Planck presented the relation describing emissivity of the black body as

$$
E_{f, T}^{\mathrm{BB}}=\frac{2 f^{2}}{c^{2}} \frac{h f}{\exp \left(\frac{h f}{k T}\right)-1},
$$

where $h$ is the Planck constant, $k$ is the Boltzmann constant, $c$ is the speed of light, $T$ is the body temperature, and $f$ is the frequency. Emissivity graph of the black body is presented in Fig. 1.

For low frequencies, i.e. at assumption that quantum energy is much lower than average energy of the $k T$ oscillator, the Planck formula takes form of the RayleighJeans relation

$$
E_{f, T}^{\mathrm{BB}} \approx \frac{2 f^{2}}{c^{2}} k T .
$$

One can see from relation (2) that for microwave band 
emissivity of the black body is directly proportional to absolute temperature of the body.

Real bodies do not have black body properties and that is why emit and absorb less energy as the black body in the same frequency range. The luminance temperature $T_{\mathrm{B}}$ of the investigated body is the temperature $T_{\mathrm{B}}$ of the black body in which its emissivity in the frequency range from $f$ to $f+\mathrm{d} f$ is equal to the emissivity $E_{f}$, of the investigated body. For microwave radiometry when the Rayleigh-Jeans approximation is valid, the luminance temperature is described by the expression

$$
T_{\mathrm{B}}=A_{f, T} T,
$$

where $A_{f, T}$ is the absorptivity of the body.

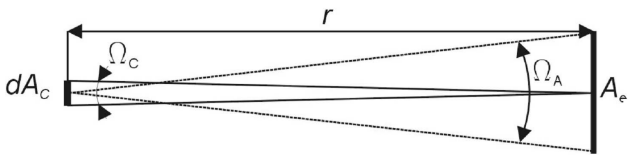

Fig. 2. Geometric relations between source and thermal radiation detector.

Geometric dependence between thermal radiation source being measured and microwave radiometer antenna is presented in Fig. 2.

For geometry shown in Fig. 2 power received by radiometer antenna in a unitary solid angle is described by the formula

$$
\mathrm{d} P_{0}=\frac{1}{2 \pi} E_{f, T}^{\mathrm{CC}} A_{\mathrm{e}} F(\Theta, \varphi) \mathrm{d} f \mathrm{~d} \Omega_{\mathrm{c}},
$$

where $\mathrm{d} \Omega_{\mathrm{c}}=\frac{\mathrm{d} A_{\mathrm{c}}}{r^{2}}$ is the unitary solid angle of the source, $F_{n}(\Theta, \varphi)$ is the normalized radiation characteristic, $A_{\mathrm{e}}$ is the effective antenna cross-section.

In order to find total power received by radiometer antenna the expression (4) should be integrated over solid angle and interval $B$ from which radiation is registered. There exists a direct relation connecting energetic luminance with thermal radiation power received by microwave receiver antenna. The total power of the black body radiation received by the antenna in the frequency interval $B$, for which we can assume that $E_{f, T}^{\mathrm{BB}}=$ const, is

$$
P=k T B .
$$

On the base of Eq. (3) the body temperature can be found via measurement of the received radiation in the microwave range.

\section{Microwave radiometer as an absolute temperature meter}

When measuring absolute temperatures of living tissues with the use of microwave radiometer, a reflection coefficient (reflectivity) at the antenna-tested tissue contact must be known. Due to very low magnetic susceptibility $\left(\mu_{\mathrm{r}} \approx 1\right)$ biological tissues are rated among lossy dielectrics. Properties of lossy dielectrics are determined by complex permittivity, [12]:

$$
\varepsilon_{t}=\varepsilon_{0}\left(\varepsilon_{\mathrm{r}}+\frac{\sigma}{\mathrm{i} \omega \varepsilon_{0}}\right)
$$

where $\varepsilon_{0}$ is the permittivity of free space, $\varepsilon_{\mathrm{r}}$ is the relative permittivity (dielectric constant) of a tissue, $\sigma$ is the tissue conductivity, and $\omega$ is the field pulsation.

It can be seen from Table in which data were taken from $[13,14]$ that electric parameters of tissues change depending on frequency and tissue type. In this connection reflection coefficient $\Gamma$ at the antenna-tested tissue contact will change, too, according to the dependence [6]:

$$
|\Gamma|^{2}=\left|\frac{\sqrt{\varepsilon_{t}}-\sqrt{\varepsilon_{\mathrm{A}}}}{\sqrt{\varepsilon_{t}}+\sqrt{\varepsilon_{\mathrm{A}}}}\right|^{2},
$$

where $\varepsilon_{\mathrm{A}}$ is the permittivity of the antenna.

Electric parameters of selected living tissues in function of the frequency.

TABLE

\begin{tabular}{l|c|c|c|c|c|c|c|c}
\hline \hline \multirow{2}{*}{ Tissue type } & \multicolumn{2}{|c|}{$0.8 \mathrm{GHz}$} & \multicolumn{2}{c|}{$1.5 \mathrm{GHz}$} & \multicolumn{2}{c|}{$2.9 \mathrm{GHz}$} & \multicolumn{2}{c}{$4.4 \mathrm{GHz}$} \\
\cline { 2 - 9 } & $\sigma$ & $\varepsilon_{\mathrm{r}}$ & $\sigma$ & $\varepsilon_{\mathrm{r}}$ & $\sigma$ & $\varepsilon_{\mathrm{r}}$ & $\sigma$ & $\varepsilon_{\mathrm{r}}$ \\
\hline body fluids & 1.61 & 68.93 & 1.87 & 68.70 & 2.86 & 67.89 & 4.56 & 66.50 \\
blood & 1.50 & 61.70 & 1.85 & 59.93 & 2.95 & 57.52 & 4.62 & 55.00 \\
kidney & 1.33 & 59.55 & 1.75 & 55.44 & 2.80 & 51.79 & 4.27 & 49.06 \\
muscles & 0.91 & 55.29 & 1.19 & 53.96 & 2.06 & 52.18 & 3.41 & 50.31 \\
dry skin & 0.83 & 41.98 & 1.07 & 39.4 & 1.69 & 37.55 & 2.61 & 36.26 \\
wet skin & 0.81 & 46.52 & 1.09 & 44.40 & 1.88 & 42.24 & 3.04 & 40.35 \\
fat & 0.049 & 5.48 & 0.068 & 5.38 & 0.125 & 5.23 & 0.206 & 5.09
\end{tabular}

Because the absorbing power $A_{f, T}$ for lossy dielectrics in the microwave range is determined by relation (8) [15]:

$$
A_{f, T}=1-|\Gamma|^{2},
$$

ence, realized via hardware using a proper radiometer scheme, is necessary to equalize the luminance temperature $T_{\mathrm{B}}$ and the physical temperature of the tested tissue.

then the compensation of the reflection coefficient influ- 


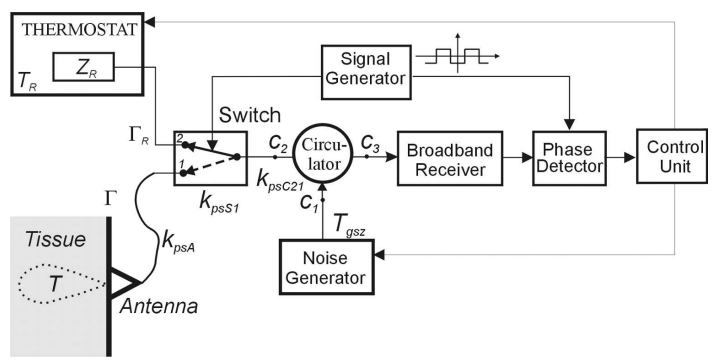

Fig. 3. Compensated microwave radiometer for absolute temperature measurement.

The block diagram of the radiometer enabling subsurface measurements of the absolute temperature of living tissues is presented in Fig. 3 while its principle of operation is described in [16]. The radiometer consists of: (a) the antenna cable, the switch, and the circulator whose transmittance coefficients are $k_{p s}$, $k_{p s S 1}$, and $k_{p s C 21}$, respectively, (b) the broadband microwave receiver with synchronous detector and control system, (c) the electronically controlled reference noise generator which power is defined by the noise temperature $T_{g s z}$, (d) the reference impedance placed in the thermostat with temperature $T_{\mathrm{R}}$ for which reflection coefficient equals $\Gamma_{\mathrm{R}}$, and (f) the antenna

$$
\begin{aligned}
& T=k_{p s A} k_{p s S 1} k_{p s C 21} T_{g s z} \\
& \quad+T_{\text {Phy }}\left(1-k_{p s A} k_{p s S 1} k_{p s C 21}\right), \\
& \Gamma_{\mathrm{R}}^{2}=k_{p s A}, \\
& T_{\mathrm{R}}=T_{\text {Phy }}-\Delta T .
\end{aligned}
$$

The absolute temperature $T$ of a tested tissue can be found from relation (9). In order to measure the absolute temperature using the radiometer shown in Fig. 3 the two conditions described by expressions (10) and (11) must be fulfilled [16, 17]. It results from the condition (10) that the thermostat temperature with reference load $Z_{\mathrm{R}}$ should be lower than the physical temperature $T_{\text {Phy }}$ of the input circuits of the radiometer ( $\Delta T$ is the difference between these temperatures). The value of $\Delta T$ depends on the input switch design of the radiometer. The value of noise temperature $T_{g s z}$ of the controlled noise source, for which voltage at radiometer phase detector output is zero, is defined with the use of the system controlling radiometer operation, while the physical temperature $T_{\text {Phy }}$ of the radiometer input circuits is measured using special sensors.

\section{Experimental results}

Radiometric measurements of the absolute temperature of the lossy medium were made after previous necessary calibration of both radiometer subassemblies and the entire radiometer. The correctness of the compensation of the influence of reflection coefficient system was verified in the measurement of noise power, originating from a non-matched resistance load with constant temperature connected to the radiometer antenna input. The results of operation of the reflection coefficient system are presented in Fig. 4.

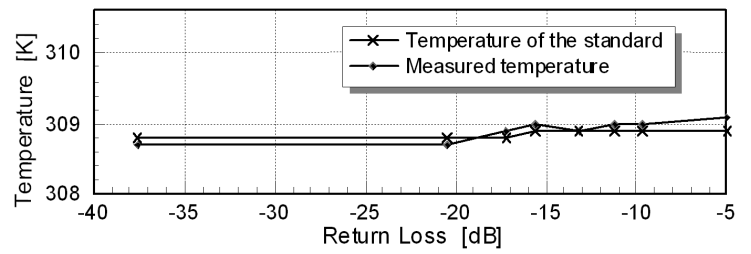

Fig. 4. Dependence of the standard temperature measured by the radiometer with reflection coefficient compensation from return loss.

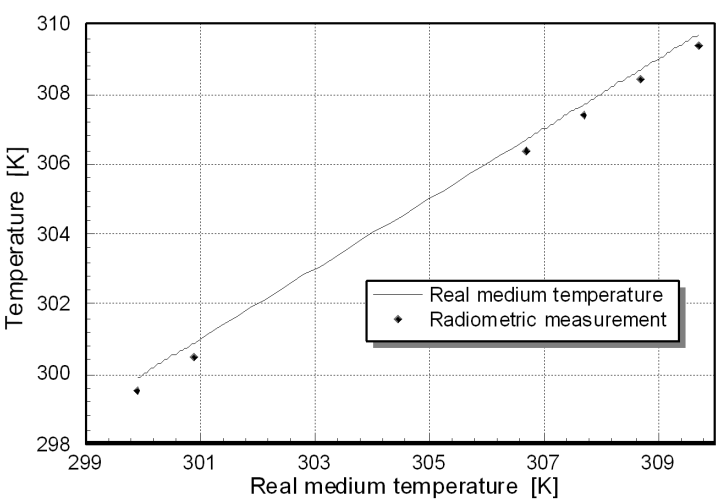

Fig. 5. Radiometric absolute temperature measurement of the homogeneous lossy medium in the form of $1 \% \mathrm{NaCl}$ water solution made in ambient temperature $T_{\text {Phy }}=294.8 \mathrm{~K}$.

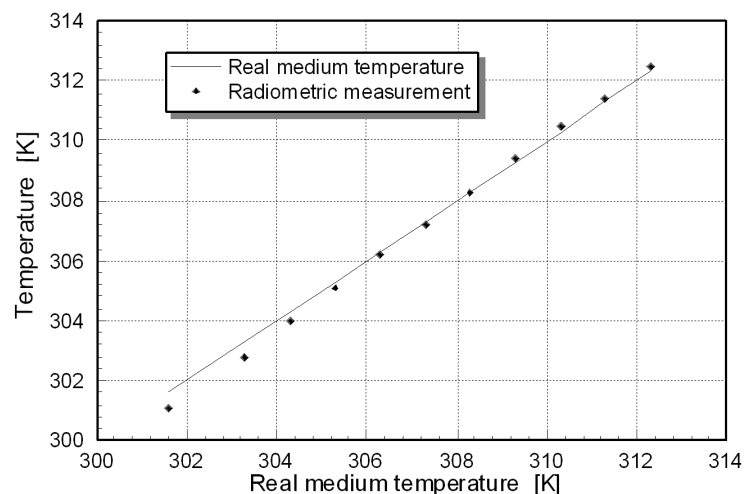

Fig. 6. Radiometric absolute temperature measurement of the homogeneous lossy medium in the form of $1 \% \mathrm{NaCl}$ water solution made in ambient temperature $T_{\text {Phy }}=(295.6 \div 296.1) \mathrm{K}$.

The next part of the paper presents measurement results of thermal radiation from a homogeneous and lossy medium, which was a $1 \%$ water solution of $\mathrm{NaCl}$. This solution was held in the local thermodynamic quasi-equilibrium defined each time for various temperatures. 


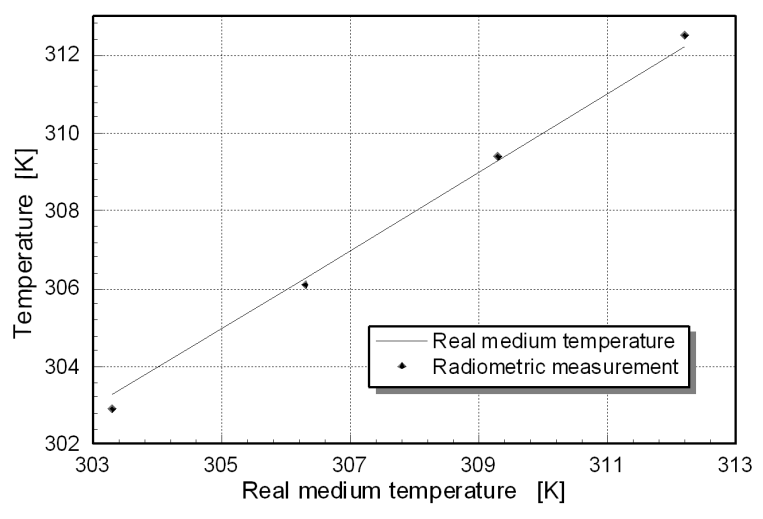

Fig. 7. Radiometric absolute temperature measurement of the homogeneous lossy medium in the form of $1 \% \mathrm{NaCl}$ water solution made in ambient temperature $T_{\text {Phy }}=296.4 \mathrm{~K}$.

The antenna used in measurement was characterized, after the contact with medium, by $-9.5 \mathrm{~dB}$ return loss. Measurements were carried out using the microwave radiometer operating at $1.5 \mathrm{GHz}$ band. In Figs. $5 \div 7$ absolute temperature measurement results are presented.

The presented measurement results confirm correctness of assumptions and performed analysis in result of which a model of microwave radiometer for absolute temperature measurement of homogeneous lossy medium in changing ambient conditions was accepted and suggested.

\section{Conclusions}

Determination of temperature of a body in radiometric measurements is related to a technique of ultraweak noise power measurements. The main task of the radiometer is determination of the antenna noise power in a situation when radiometer internal noise depend on the same probabilistic distributions as measured signal and are on the comparable energy level. In the literature devoted to radiometry and microwave thermography applications in medical diagnostics, there are presented problems related to measurements of differences in temperatures of adjacent tissues, i.e. relative measurements.

In the paper the solution of absolute measurements is presented. The measured thermal radiation is emitted by living and functioning organism. Occurrence of living processes in the organism makes unambiguous determination of reference temperature for relative measurements difficult. In any part of tissue being measured, temperature differences can be very small, even if the internal area of a tissue can be in pathological condition, which is characterized by elevated temperature. In that case determination of a pathological condition of a tissue on the base of temperature difference value between its fragments seems to be hardly reliable. Moreover, relative measurements need constant ambient parameters during the whole measurement cycle, which for long- -lasting measurements is very difficult to maintain without special procedures (like for example air-conditioned or isothermal rooms). This limits the use of radiometers only for preliminary and screening examinations, which could and should be carried out quickly and in various environmental conditions. For the presented microwave radiometer the uncertainty of absolute temperature measurement was determined analytically. For the $68.27 \%$ confidence level the absolute temperature value measured by the microwave radiometer with compensation of the influence of reflection coefficient is located in the $(T-0.55, T+0.55) \mathrm{K}$ interval, while for $99.73 \%$ confidence level - in the range of $(T-1.65, T+1.65) \mathrm{K}$.

\section{References}

[1] G. Evans, C.W. McLeish, R.F. Radiometer Handbook, Artech House, Norwood 1977.

[2] M. Chive, Methods of Hyperthermia Control, Berlin 1987, p. 113.

[3] L. Dubois, J. Pribetich, J. Falbre, M. Chive, Y. Moschetto, Int. J. Hyperthermia 9, 193 (1993).

[4] F. Bardati, G. Marrocco, P. Tognolatti, IEEE Trans. MTT 52, 1167 (2004).

[5] K. Maruyma, S. Mizushina, T. Sugiura, G.M.J. Van Leeuwen, J.W. Hand, G. Marrocco, F. Bardati, A.D. Edwards, D. Azzopardi, D. Land, IEEE Trans. MTT 48, 11, 995 (2000).

[6] B. Stec, Aparatura Naukowo-Dydaktyczna 4, 21 (1990) (in Polish).

[7] B. Przywara-Chowaniec, L. Poloński, M. Gawlikowski, T. Pustelny, Acta Phys. Pol. A 116, 344 (2009).

[8] G. Konieczny, Z. Opilski, T. Pustelny, E. Maciak, T. Pustelny, Acta Phys. Pol. A 116, 389 (2009).

[9] B. Bocquet, J.C. Van De Velde, A. Mamouni, Y. Leroy, G. Giaux, J. Delannoy, D. Delvalee, IEEE Trans. MTT 38, 491 (1990).

[10] W. Kazanowska, P. Knapp, A. Mazurek, T. Filipkowski, B. Stec, in: 8th Int. Meeting of Gynaecological Oncology, Barcelona 1993, p. 73.

[11] P. Knapp, A. Mazurek, M.J. Puciłowska, W. Kazanowska, B. Stec, in: Polish Oncology. The XXV Jubilee Meeting of Polish Gynaecological Association, 1994, p. 1598 (in Polish).

[12] J. Szóstka, Waves and Antennas, WKŁ, Warszawa 2000 (in Polish).

[13] S. Gabriel, R.W. Lau, C. Gabriel, Phys. Med. Biol. 41, 163 (1996).

[14] http://niremf.ifac.cnr.it, Italian National Research Council, Institute for Applied Physics, Florence, Italy.

[15] K.M. Ludeke, J. Kohler, J. Microwave Power 18, 223 (1993).

[16] B. Stec, M. Żurawski, in: 23rd European Microwave Conf., Madrid, 1993, p. 269.

[17] W. Susek, Ph.D. Thesis, Military University of Technology, Warszawa 2005 (in Polish). 